ENCYCLOPÉDIE Encyclopédie berbère

BERBERE

3 | 1986

3 | Ahaggar - Alī ben Ghaniya

\title{
Aïn Dokkara
}

\section{M.-C. Chamla et J. Dastugue}

\section{OpenEdition}

Journals

Édition électronique

URL : http://journals.openedition.org/encyclopedieberbere/834

DOI : $10.4000 /$ encyclopedieberbere.834

ISSN : 2262-7197

\section{Éditeur}

Peeters Publishers

\section{Édition imprimée}

Date de publication : 1 juillet 1986

Pagination : 326-330

ISBN : 2-85744-260-2

ISSN : 1015-7344

\section{Référence électronique}

M.-C. Chamla et J. Dastugue, «Aïn Dokkara », Encyclopédie berbère [En ligne], 3 | 1986, document A116, mis en ligne le 01 décembre 2012, consulté le 12 octobre 2020. URL : http://journals.openedition.org/ encyclopedieberbere/834; DOI : https://doi.org/10.4000/encyclopedieberbere.834

Ce document a été généré automatiquement le 12 octobre 2020

(c) Tous droits réservés 


\title{
Aïn Dokkara
}

\author{
M.-C. Chamla et J. Dastugue
}

1 Gisement préhistorique du type escargotière situé sur un éperon dominant le ravin de l'Aïn Dokkara, à l'est de Tébessa et à $5 \mathrm{~km}$ à vol d'oiseau de la frontière tunisienne, près du col de Bekkaria. Découverte dès 1934, cette escargotière, nommée aussi " escargotière du chacal », fut fouillée par L. Balout et E. Sérée de Roch qui dégagèrent en 1949 un squelette d'homme capsien à la base d'une tranchée qu'ils avaient ouverte dans la masse du gisement (L. Balout, 1949).

2 L'industrie lithique recueillie est celle d'un Capsien supérieur du type d'Aïn Aachena (J. Tixier 1976), remarquable par la qualité du débitage par pression, l'élégance de ses lamelles et de ses microlithes géométriques. Une des datations radiométriques faite à partir d'échantillons de coquilles d'hélix recueillies à la partie inférieure du gisement correspondant au niveau d'où fut extrait le squelette, indiquent un âge de $6580 \pm 100$ $\mathrm{BC}$.

3 Le squelette était étendu orienté est-ouest en décubitus latéral droit fléchi, face contre terre au contact d'une pierre brûlée placée sous la joue droite. 


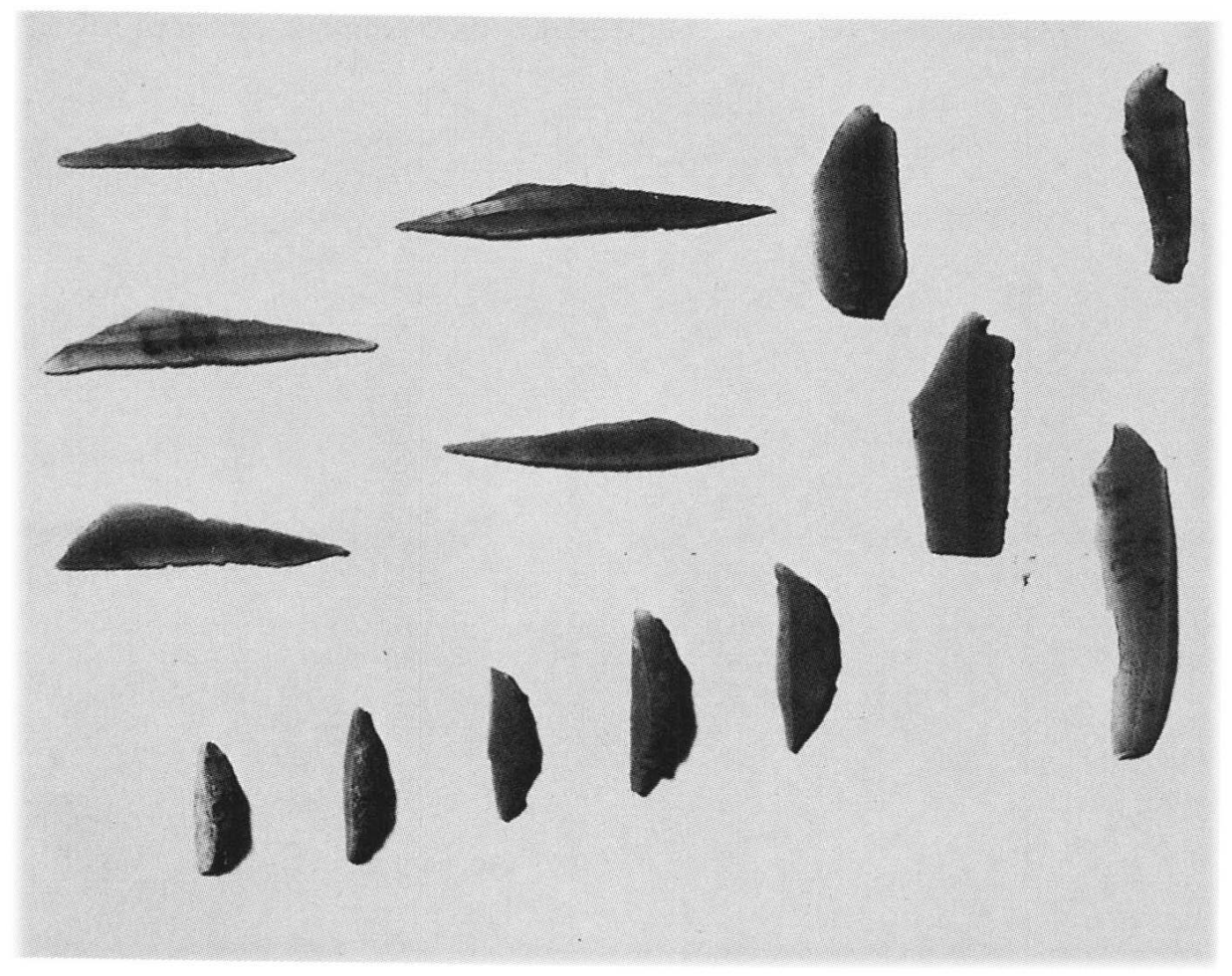

4 Restaurés au laboratoire du Musée du Bardo à Alger, ces restes sont ceux d'un individu dont le squelette, presque complet, était fort bien conservé.

D'après le type général du crâne dont l'aspect est assez robuste, les apophyses mastoïdes longues et vigoureuses, le relief de la région iniaque assez développé, les arcades sus-orbitaires plutôt saillantes, la forme du menton triangulaire, ainsi que le faible degré d'abrasion de la denture, il s'agit d'un home adulte jeune, âgé d'environ 25 à 30 ans.

6 Par l'ensemble de ses caractères, notamment sa dolichocrânie, sa face de hauteur moyenne et assez étroite, ses orbites carrées, son nez de largeur moyenne, son prognathisme alvéolaire allié à un orthognathisme facial, ses grandes dimensions crâniennes, ses reliefs faciaux assez marqués, sa stature élevée, ses proportions corporelles, l'Homme de l'Aïn Dokkara se rattache au type protoméditerranéen (forme robuste). Celui-ci paraît comporter en Afrique du Nord à l'époque épipaléolithique deux variantes ou sous-types que l'on rencontre essentiellement dans les escargotières de la régión orientale de l'Algérie et qui sont les suivantes :

7 1. Un type dolichocrâne à mésocrâne, à voûte haute ou moyenne, à face longue, aux orbites méso- à hypsiconques, au nez méso-leptorhinien, à stature élevée, qui caractérise notamment les hommes des gisements de Gambetta ( $n^{\circ} 1$, d'époque ibéromaurusienne incertaine), d'Aïoun Beriche, de Medjez II ( $n^{\circ} 1$ et 3), d'Aïn Méterchem (néolithique ?), et la femme de Khanguet-el-Mouhaad.

2. Un type dolichocrâne à mésocrâne, à voûte basse, à face moyenne, aux orbites mésoconques, au nez mésorhinien, à stature élevée dont l'Homme de l'Aïn Dokkara peut être considéré comme un représentant typique, ainsi que les deux femmes trouvées dans les gisements de Rachgoun (ibéromaurusien) et de Medjez II ( $\left.n^{\circ} 2\right)$. 
Dans l'état actuel de nos connaissances, le premier type semble davantage représenté en Afrique du Nord que le second dans les gisements capsiens.

Ces Protoméditerranéens apparaissent fondamentalement différents des Hommes épipaléolithiques du type de Mechta-Afalou d'Afrique du Nord. La comparaison entre le squelette de l'Homme de l'Aïn Dokkara avec les restes provenant des gisements ibéromaurusiens d'Afalou (Algérie) et de Taforalt (Maroc) montre effectivement une assez grande dissemblance bien que l'on note plusieurs dispositions spéciales communes qui présentent un certain intérêt car elles indiquent qu'il existait malgré tout des affinités entre les deux types. Une partie d'entre elles paraît être commune aux autres Hommes préhistoriques mésolithiques d'Europe et du Proche-Orient, alors que d'autres semblent être propres aux Hommes vivant dans cette région de l'Afrique.

11 Les différences essentielles entre les Hommes de Mechta-Afalou et l'Homme protoméditerranéen de l'Aïn Dokkara consistent en une robustesse générale moins grande de ce dernier, des dimensions moins considérables, une stature moins élevée, des reliefs osseux moins développés et une morphologie crânio-faciale moins archaïque et moins dysharmonique que celle des premiers. Sa voûte crânienne, moins élevée, est munie de sutures plus sinueuses, son développement sus-orbitaire est moins marqué, sa face moins basse et moins large, ses orbites plus hautes, son nez plus étroit. Sur sa mandibule on ne constate aucune extroversion des gonions, sa branche horizontale est plus mince, ses branches montantes plus grandes et plus redressées. Absence de mutilation et de lésions pathologiques dentaires, abrasion des dents plus faibles, sont également des caractéristiques qui éloignent l'Homme de l'Aïn Dokkara des Hommes de Mechta-Afalou.

\section{Crâne de l'Homme d'Aïn Dokkara (photo Delorme)}

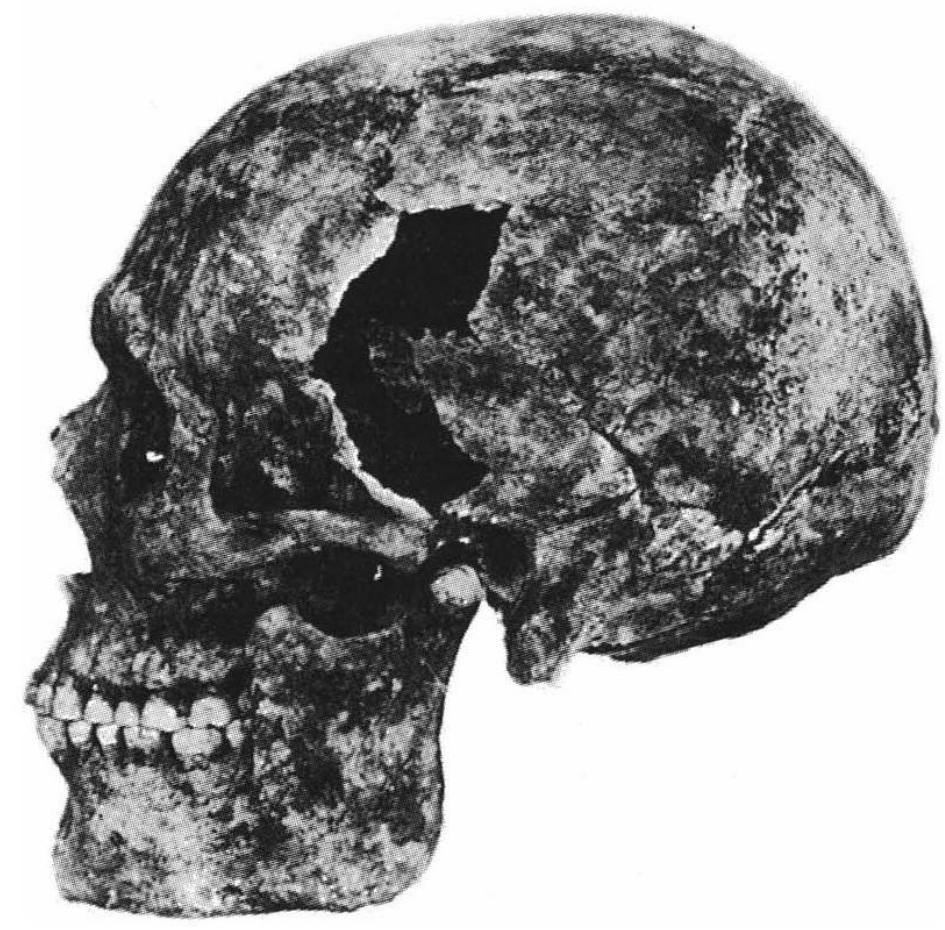



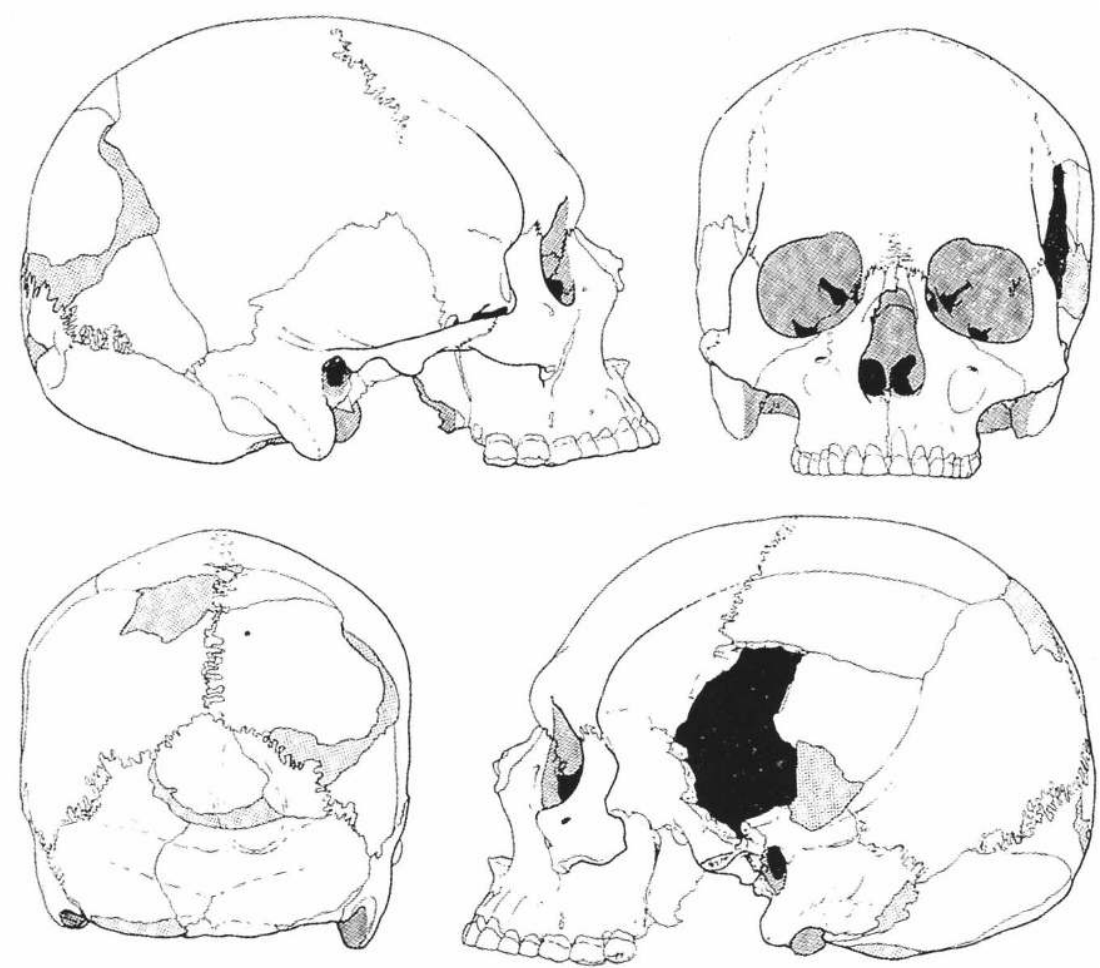

Principales caractéristiques métriques de l'Homme de l'Aïn Dokkara

Signalement

Capacité crânienne 1524,4 cc aristencéphale

Ind. crânien 71,5 dolichocrâne

Ind. moyen de hauteur 80,4 hauteur moyenne

Ind. frontal-transversal 84 moyennement divergent

Ind. facial supérieur 52,7 mésène

Ind. crânio-facial transversal 98,2 crypto-phénozyge

Ind. orbitaire 83,3 mésoconque

Ind. nasal 48,9 mésorhinien

Ind. palatin 79,6 leptostaphylin

Angle prognathisme alvéolaire $71^{\circ}$ prognathe

Stature 1,70 m environ assez élevée

M.-CL. CHAMLA

\section{Pathologie}

Le seul stigmate pathologique digne d'être noté sur ce squelette est un petit renfoncement, très localisé, de la paroi crânienne.

La lésion siège sur l'écaille de l'occipital, à $1 \mathrm{~cm}$ à droite de la ligne médiane, à $1 \mathrm{~cm}$ audessus de la ligne courbe supérieure. C'est une dépression en forme de sillon semicirculaire dont la concavité regarde vers le haut. Large d'environ $2 \mathrm{~mm}$ en son milieu, ce sillon s'évase à ses deux extrémités ; sa longueur totale est de $30 \mathrm{~mm}$. 
15 A la dépression de la table externe, ci-dessus décrite, correspond exactement, sur la table interne, une saillie en forme de bourrelet. La nature de la lésion ne peut donc faire aucun doute: il s'agit d'un enfoncement traumatique. Le déplacement du fragment endocrânien est minime, la dénivellation étant au maximum de $3 \mathrm{~mm}$. Par ailleurs, la plage traumatisée est très réduite en sorte qu'on peut conclure, sans trop s'avancer, que ses conséquences ont dû être bénignes.

Malgré ce faible degré d'enfoncement et cette exiguïté de la plage traumatique, une telle lésion supposerait, chez un adulte, un impact assez violent pour fracturer l'os sur toute son épaisseur et assez limité en amplitude pour ne pas se poursuivre en profondeur et causer l'éclatement de la table interne. Cela n'est certes pas impossible mais on ne peut s'empêcher de penser que la réalisation d'un tel traumatisme doit être bien plus facile sur la mince écaille occipitale d'un enfant. L'hypothèse d'une blessure infligée dans le jeune âge, sans pouvoir être effectivement prouvée, paraît néanmoins la plus vraisemblable.

\section{BIBLIOGRAPHIE}

ARAMBOURG C, BOULE M., VALLOIS H. et VERNEAU R. Les grottes paléolithiques des Beni-Segoual (Algérie). - Arch. Inst. Paléont. hum., n 13, 1934, 242 p.

BALOUT L. Découverte d'un squelette humain préhistorique dans la région de Tébessa. Bull. Sté d'Hist. nat. d'Afrique du N. 1949, t. 40, p. 193-195.

- Les Hommes préhistoriques du Maghreb et du Sahara. Inventaire descriptif et critique (Paléolithique, Epipaléolithique, Néolithique). Libyca, Anthrop. Préhist. Ethnogr., 1953, t. 1, pp. 120-140.

BALOUT L. et ROUBET C. Datation radiométrique de l'Homme capsien de l'Aïn Dokkara et de son gisement (« escargotière du Chacal », région de Tébessa, Algérie), Libyca, 1970, t. 18, p. 23-34. BRIGGS L.-C. Tête osseuse du Khanguet-el-Mouhaad (fouilles J. Morel). Libyca, Anthrop. Préhist. Ethnogr., 1953, t. 1, pp. 120-140.

CAMPS G. Les Civilisations préhistoriques de l'Afrique du Nord et du Sahara. Doin 1974, 376 p.

CAMPS FABRER H. Un gisement du Capsien supérieur. L'escargotière de Medjez II (El Eulma), département de Sétif, Algérie. Note préliminaire. L’Anthropologie, 1968, t. 72, n 5-6, pp. 479-488.

CAMPS-FABRER H. Un gisement capsien de faciès sétifien. Medjez II. El Eulma (Algérie) C.N.R.S., Paris, 1975, $448 \mathrm{p}$.

CHAMLA M.-C. Note sur les restes humains (H 4) découverts à Rachgoun en Février 1964. Libyca Anthrop. Préhist. Ethnogr., 1966, t. 14, pp. 182-188.

- Étude anthropologique de l'Homme capsien de l'Aïn Dokkara, Libyca, Anthrop. Préhist. Ethnogr., 1973, t. XXI, pp. 9-50. 
- Les Hommes épipaléolithiques de Columnata (Algérie occidentale). Étude anthropologique. Mém. Centre rech, anthrop. préhist. ethnogr., Alger, t. XV, 1970, 132 p. (avec la collaboration de J.-N. BIRABEN et J. DASTUGUE).

DASTUGUE J. Note sur la pathologie de l'homme de l'Aïn Dokkara. Libyca, Anthrop. Préhist. Ethnogr., 1973, t. XXI, p. 51.

FEREMBACH D. La nécropole épipaléolithique de Taforalt (Maroc oriental). Étude des squelettes humains. Casablanca, 1962, $175 \mathrm{p}$.

TIXIER J. L'industrie lithique capsienne de l'Aïn Dokkara. Libyca, t. XXI, 1976, pp. 21-54.

VAlloIS H. Le squelette d'Aïn Meterchem. Atti del I Congr. int. di Preistor. Mediterr., Florence, 1950, pp. 102-104.

INDEX

Mots-clés : Algérie, Anthropologie, Préhistoire 